\title{
Macro-Operators in Multirelational Learning: A Search-Space Reduction Technique
}

\author{
Lourdes Peña Castillo and Stefan Wrobel \\ Otto-von-Guericke-University Magdeburg \\ \{pena, wrobel\}@iws.cs.uni-magdeburg.de
}

\begin{abstract}
Refinement operators are frequently used in the area of multirelational learning (Inductive Logic Programming, ILP) in order to search systematically through a generality order on clauses for a correct theory. Only the clauses reachable by a finite number of applications of a refinement operator are considered by a learning system using this refinement operator; ie. the refinement operator determines the search space of the system. For efficiency reasons, we would like a refinement operator to compute the smallest set of clauses necessary to find a correct theory. In this paper we present a formal method based on macro-operators to reduce the search space defined by a downward refinement operator $(\rho)$ while finding the same theory as the original operator. Basically we define a refinement operator which adds to a clause not only single-literals but also automatically created sequences of literals (macro-operators). This in turn allows us to discard clauses which do not belong to a correct theory. Experimental results show that this technique significantly reduces the search-space and thus accelerates the learning process.
\end{abstract}

\section{Introduction}

Typically, a multirelational learning system takes as input background knowledge $B$, positive examples $E^{+}$and negative examples $E^{-}$, and has to find a correct theory $T$. A correct theory is a set of Horn clauses which implies $E^{+}$and is consistent ${ }^{1}$ with respect to $E^{-}$. This theory is then used to classify unseen examples $E^{\text {? }}$ as positive or negative. To find $T$ the system has to search among permitted clauses (hypothesis space) for a set of clauses with the required properties. For instance, if a $e \in E^{+}$is not implied by $T$ the system should search for a more general theory; on the other hand, if $T$ is not consistent with $E^{-}$the system should look for a more specific one.

Refinement operators are commonly used in multirelational learning systems as a way to systematically search for $T$. A refinement operator is a function which computes either a set of specializations (downward operator) or generalizations (upward operator) of a clause. Thus, refinement operators allow us to search

\footnotetext{
${ }^{1}$ Note, however, that the condition of consistency is often relaxed and the systems actually try to minimize the classification error over the training data.
} 
step-by-step through a generality order ${ }^{2}$ on clauses for a correct theory. The search space $H$ is then restricted to clauses that can be obtained by successively applying the refinement operator. This approach works if and only if there is a number of refinement steps to every clause in at least one correct theory. [8]

For efficiency reasons, we would like a refinement operator $\rho$ to compute the smallest set of clauses necessary to find $T$. Many downward operators currently used work by adding to a clause $C$ one literal available from $B$. In this paper we present a method based on automatically created macro-operators [5] to reduce the hypothesis space defined by one of these refinement operators. In the search and planning literature a macro-operator (or macro for short) is a sequence of operators chosen from the primitive "operators" of a problem. We consider our primitive operators the literals available to be added to $C$, so a macro is a sequence of literals. By refining $C$ using only "valid" literals and macros, we discard a significant number of clauses (hypotheses) which cannot belong to $T$.

Macros are based on the fact that literals exist which if added alone to a clause $C$ affect neither the coverage nor the consistency of $C$. For example, consider we want a system to learn the concept divisibleBy $(x, y)$ ( $x$ is divisible by $y$ ) and as part of $B$ we give the literals $i s N u l l(z)$ and remainder $(x, y, z)$ where $x, y$ are input arguments and $z$ is computed by remainder and used by is Null. The literal remainder is true for every combination of numeric values for $x$ and $y$ and adding it by itself to $C$ does not help to distinguish between $E^{+}$ and $E^{-}$. However, the macro remainder $(x, y, z), i s N u l l(z)$ is certainly more useful; thus our $\rho$ adds to $C$ this macro instead of adding either remainder alone or remainder together with another literal that does not use $z$.

In this paper, we precisely develop the macro-based approach for the class of refinement operators that are lower bounded by a bottom clause, such as the one used in Progol [7]. Specifically, we adapt the refinement operator used in Mio [9] (henceforth referred to as literal-based $\rho$ ). The literal-based $\rho$ is then used in our experiments as our comparison point. Experiments on four application domains show that the reduction of the search space obtained using the macro-based $\rho$ produces a significant speed-up of the learning process. Using the macro-based $\rho$ implies to the user almost no extra effort since: 1) a formal characterization of literals which helps to decide whether a literal can be included in a macro without risk of missing a solution is provided, and 2) the mode declaration language is enhanced to allow the user to easily declare these literals. In addition, the macros are domain-independent and constructed automatically by the system based on the mode declarations given by the user.

The remaining of this paper is organized as follows. The next section describes the learning system used and the literal-based $\rho$. Section 3 explains how the search space reduction is achieved. Section 4 defines a macro, the macro-based $\rho$ and the macro generation algorithms. Section 5 discusses our experiments. Related work is briefly surveyed in Section 6 and Section 7 concludes.

\footnotetext{
${ }^{2}$ A generality order determines what constitutes a "specialization" or "generalization" of a clause. Subsumption and logical implication are two of the most widely used generality orders.
} 


\section{Mio and the Literal-Based $\rho$}

\subsection{The Learning System}

Mio is an example-driven learning system introduced in [9] which uses a Progollike declaration language and, the same as Progol [7], lower bounds the search space with a most specific clause $\perp$. This $\perp$ is a minimal generalization sufficient to cover an example $e \in E^{+}$. Mio performs a general-to-specific IDA* search of the hypothesis space to find a clause $C$ to add to $T$. IDA* is guided by the number of literals needed to obtain an I/O-complete clause. An I/O-complete clause has neither unbound output variables in the head nor unbound input variables in the body. In addition, Mio (contrary to Progol) selects stochastically the examples from which it learns, performs parallel search and enforces type strictness.

To construct $\perp$, the user has to give a set of mode declarations. These mode declarations define the literals from $B$ which can be used as the head and in the body of the clauses in $H$. In the mode declarations the arguments of a literal are defined either as a constant (\#cte), as an output variable (-var), or as an input variable (+var). A literal provides a value if it has a -var and consumes a value if it has a + var. We can say that a literal $p$ is a consumer of literal $q$ if $p$ has at least one +var bound to an output argument value of $q$ ( $q$ is then a provider of $p$ ). A literal $p$ defined by the mode declarations can appear in $\perp$ if and only if $p$ has at least one provider placed before $p$ in $\perp$ for each $+v a r \in p$. The most specific clause is then defined as follows.

Definition 1 (Most Specific Clause $\perp[7])$. Let $\perp$ be the most specific definite clause (bottom clause) constructed with the literals defined by the mode declarations, background knowledge $B$ and example $e \in E^{+}$such that:

(a) $B \wedge \perp \vdash_{h}$ e (ie. e can be derived in $h$ resolution steps).

(b) $\perp_{\infty} \succeq \perp$ where $\perp_{\infty}$ is the (potentially infinite) conjunction of ground literals which are true in all models of $B \wedge \neg E$ (ie. $B \wedge \neg E \models \perp_{\infty}$ ).

The most specific clause can be seen as a sequence of literals where every literal is uniquely mapped to its position in $\perp$ starting with the first body literal after the head of $\perp$ (ie. $h \leftarrow 1, \ldots, i-1, i, \ldots, n$.). We refer henceforth to every literal $i(1 \leq i \leq n)$ in $\perp$ as the integer corresponding to its position. The literals in $\perp$ are placed according to their consumer-provider relationships.

For both the literal-based and the macro-based method, we assume that the clauses are evaluated by a heuristic function $\operatorname{eval}(C)$ considering the length of $C$ $(|C|=$ number of body literals in $C$ ) and the number of positive $p$ and negative $n$ examples covered by $C$, and that $\operatorname{eval}(C)^{3}$ favours shorter clauses over longer ones with the same coverage and consistency.

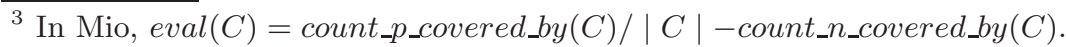




\section{$2.2 \quad$ Literal-Based $\rho$}

The literal-based refinement operator consists in adding one literal from $\perp$ to a clause $C$. The search space $H$ defined by this operator allows the system to search from the empty clause $(\square)$ to $\perp$. Notice that by the definition of $\rho$ given below the hypotheses' literals keep the topological order of $\perp$.

Definition 2 (Literal-Based $\rho$ ). Let $i$ and $j$ be literals in $\perp$ and $C$ be a clause whose last literal is $i$, then:

$$
\rho(C)=\{C \cup\{j\} \mid
$$

$i<j$ and all + var $\in j$ are either bound to -var of literals already in $C$ or to +var in the head of $C\}$.

Assume $C^{\prime} \in \rho(C)$, then $C^{\prime}$ is heuristically evaluated $\left(\operatorname{eval}\left(C^{\prime}\right)\right)$ iff $C^{\prime}$ is $\mathrm{I} / \mathrm{O}$-complete. Then, the search space is the following.

Definition 3 (Hypothesis Space $H$ ). The hypothesis space $H$ consists of I/O-complete hypotheses whose literals appear in $\perp$ :

$$
H:=\{C \subseteq \perp \mid C \text { is I/O-complete }\} .
$$

Mio considers a clause $C \in H$ as a solution (ie. as a candidate to be included in $T$ ) iff $C$ maximizes $\operatorname{eval}(C)$ and satisfies several user-defined parameters such as maximum length allowed, minimum required coverage per clause, etc.

Lemma 1. Let $C^{\prime} \in \rho^{j}\left(C_{j}\right)$. $C^{\prime}$ can be a solution iff there is no $C \in \rho^{i}\left(C_{i}\right), i<j$ $\operatorname{such}$ that $\operatorname{eval}(C) \geq \operatorname{eval}\left(C^{\prime}\right)$.

\section{Reducing the Search Space}

As explained in the introduction sometimes there are literals defined in the mode declarations that, when queried, succeed for each combination of input argument values. These literals usually are needed to introduce a variable. The $\rho$ described in Def. 2 produces clauses $C^{\prime} \in \rho(C)$ which might vary from $C$ only in having one of these "non-gain" ${ }^{4}$ literals added. However, since these literals succeed for every combination of input argument values, they modify neither the coverage nor the consistency of $C$ but they do increase its length (ie. $\left.\operatorname{eval}(C) \geq \operatorname{eval}\left(C^{\prime}\right)\right)$. Thus $C^{\prime}$ cannot be a solution and could be safely removed from $H$.

One way to avoid that $C^{\prime}$ differs from $C$ only in having one of these "nongain" literals added is to identify such literals in the mode declarations and to use a $\rho$ which does not add "non-gain" literals by themselves to $C$ but only as part of a macro. Since these "non-gain" literals usually contain output variables, we can add them to $C$ together with at least one of their consumers. To declare the "non-gain" literals, we enhance the mode declaration language with the *var notation. A *var is an output variable argument of a "non-gain" literal. To decide whether a literal should not be added by itself to $C$, we divide the literals in the following categories based on their consumption/providing properties.

\footnotetext{
${ }^{4}$ The information gain of using this literal is null.
} 
Definition 4. Let $i$ be a literal in the body of $\perp$. Then:

1. $i$ is called an independent provider iff $i$ has output variables but does not succeed for every combination of input argument values (it does not contain *var). (e.g. see literals $b$ and $c$ in Table 1)

2. $i$ is called a dependent provider iff $i$ has output variables and succeeds for every combination of input argument values (it contains at least one $* v a r$ ). (e.g. see literal a in Table 1)

3. $i$ is called a head provider iff $a$-var in the head of $\perp$ is bound to an output variable (-var or *var) in $i$. (e.g. see literals $b$ and $e$ in Table 1)

4. $i$ is called an independent consumer iff each of its +var is bound to a +var in the head of $\perp$ or to a-var in a provider. (e.g. see literal e in Table 1)

5. $i$ is called a dependent consumer iff at least one of its +var is only provided by *var (it is not bound to a +var in the head of $\perp$ or to a -var in a provider). (e.g. see literal d in Table 1)

To illustrate the literal characterization given above, assume we want to learn the target predicate $h(+x,+y,-u)$ and we are given Table 1 . Since $a$ is satisfied by all the examples and has an output variable, $a$ is a dependent provider. On the other side $a$ is the only provider of $d$, thus $d$ is a dependent consumer. In the mode declarations $a$ has to be declared as $a(+x, * w)$. All the other mode declarations are identical to the original literal definitions. Table 2 shows how a literal has to be used based on its classification. If a literal is not a provider one should ignore the first column.

Theorem 1. Let $i$ be a dependent provider in $C^{\prime} \in \rho(C) . C^{\prime}$ is not a solution if there is not at least one consumer of $i$ in $C^{\prime}$ too.

Proof (by contradiction). Assume that $C^{\prime}=C \cup\{i\}$ is a solution and $i$ is a dependent provider and there is not a consumer of $i$ in $C^{\prime}$. But, since $i$ succeeds for every combination of input argument values, $\operatorname{eval}(C) \geq \operatorname{eval}\left(C^{\prime}\right)$ and since $C^{\prime}=C \cup\{i\},|C|<\left|C^{\prime}\right|$. Hence $C^{\prime}$ cannot be a solution (Lemma 1) which contradicts the assumption and completes the proof.

By Definition 4 and Theorem 1 we can then conclude that the search space can be further restricted to $H^{\prime}:=\{C \subseteq \perp \mid C$ is I/O-complete and each depen-

Table 1. Toy example to illustrate the classification of literals

\begin{tabular}{|l||c|c|c|}
\hline Literal Definition & Instance & $\mathbf{h}\left(\mathbf{I}_{\mathbf{i}}\right)$ & Satisfied Literals \\
\hline$h(+x,+y,-u)$ & $I_{1}$ & + & $a, b, d, e$ \\
\hline$a(+x,-w)$ & $I_{2}$ & + & $a, c, d, e$ \\
\hline$b(+y,-u,-z)$ & $I_{3}$ & + & $a, b, d$ \\
\hline$c(+x,+y,-z)$ & $I_{4}$ & - & $a, c, e$ \\
\hline$d(+w)$ & \multicolumn{3}{|l}{} \\
\hline$e(+z,-u)$ & \multicolumn{3}{|l}{} \\
\hline
\end{tabular}


Table 2. Literal use according to its classification

\begin{tabular}{|c|c|c|}
\hline Provider Type & Consumer Type & Literal Use \\
\hline \hline Independent-Provider & Independent-Consumer & As a single literal \\
\hline Independent-Provider & Dependent-Consumer & In macros \\
\hline Dependent-Provider & - & In macros \\
\hline Head-Provider & - & As a single literal \\
\hline
\end{tabular}

dent provider in $C$ has at least one consumer for one of its $* v a r\}$. In the next section we propose a refinement operator to accomplish this reduction.

\section{Macro-Based Method}

So far we have intuitively used the term macro in relation to a refinement operator, but now we are ready to formally define it. For that, we define first what a legal subsequence of literals is.

Definition 5 (Subsequence of Literals). Let $\perp$ be a sequence of literals $\perp=$ $1 \ldots n$ then $j_{1} \ldots j_{i}(i \leq n)$ is a subsequence of literals of $\perp$ when $j_{k}<j_{k+1}, \forall k \in$ $\{1, \ldots, i-1\}$ and $j_{k} \in[1, n], \forall k \in\{1, \ldots, i\}$.

Definition 6 (Legal Subsequence of Literals Given Another Subsequence). Let $j_{1} \ldots j_{i-1}$ be a legal subsequence of literals according with Def. $\%$. Then $j_{i} \ldots j_{m}(m \leq n)$ is a legal subsequence of literals given $j_{1} \ldots j_{i-1}$ iff:

(a) every $*$ var of dependent-provider $j_{i}$ is used by a consumer $\in\left\{j_{i+1}, \ldots, j_{m}\right\}$ or $j_{i}$ is also a head-provider; and,

(b) every +var of consumer $j_{s}, j_{i}<j_{s} \leq j_{m}$, is bound to an output variable in a provider $\in\left\{j_{1}, \ldots, j_{s-1}\right\}$ or to a +var in the head of $\perp$.

Definition 7 (Legal Subsequence of Literals). A subsequence of literals $j_{1} \ldots j_{i-1}$ is a legal subsequence iff $j_{1} \ldots j_{i-1}$ is a legal subsequence given $\emptyset . \emptyset$ is also a legal subsequence.

Definition 8 (Macro-Operator). A macro-operator is the shortest legal subsequence of literals $j_{i} \ldots j_{m}$ for which exists a subsequence $j_{1} \ldots j_{i-1}$ of literals in $\perp$ where $j_{g}<j_{i}, \forall g \in\{1, \ldots, i-1\}$, so that $j_{i} \ldots j_{m}$ is legal given $j_{1} \ldots j_{i-1}$.

\subsection{Macros' Ordering}

In the literal-based method the literals are ordered by their position in $\perp$. For the macro-based method we introduce a new ordering based on the maximum provider of the literals in a macro. 
Definition 9 (Maximum Provider of a Literal). Let $j$ be a consumer. The maximum provider of $j$ is provider $i$ of $j(i<j)$ with the greatest position in $\perp$. The position of the head of $\perp$ is 0 .

Definition 10 (Maximum Provider of a Subsequence of Literals). The maximum provider of a subsequence of literals $a=j_{i} \ldots j_{m}$ is:

$$
\max \_p r o v(a)=\max _{j_{k} \in\left\{j_{i} \ldots j_{m}\right\}}\left(\text { maximum_provider_of_literal }\left(j_{k}\right)\right)
$$

Definition 11 (Comparison between Subsequences of Literals). Let $a=$ $j_{i} \ldots j_{m}$ and $b=j_{k} \ldots j_{n}$ be legal subsequences of literals, then $a<b$ iff:

(a) max_prov $(a)<$ max_prov(b); or,

(b) max_prov $(a)=$ max_prov(b) and a is lexicographically $<b$ (ie. $j_{i}<j_{k} \wedge$ $\left.j_{i+1}<j_{k+1} \wedge \ldots \wedge j_{m}<j_{k+m}\right)$.

\subsection{Macro-Based Refinement Operator}

Now everything is ready to define the macro-based refinement operator. Note that single literals can also fulfill the macro's definition and be used in the refinement operator defined below.

Definition 12 (Macro-Based Refinement Operator $\rho^{\prime}$ ). Let $a$ and $b$ be macros obtained from $\perp$ and $C$ be a clause (and a legal subsequence of literals) whose last added macro is a, then:

$$
\rho^{\prime}(C)=\{C \cup\{b\} \mid
$$

$b \geq a$ and $b$ is a legal subsequence given $C\}$.

Assume $C^{\prime} \in \rho^{\prime}(C)$, then $C^{\prime}$ is heuristically evaluated iff $C^{\prime}$ is I/O-complete.

We now prove the crucial property of the macro-based approach, namely that the macro-based $\rho$ finds the same solutions as the literal-based one.

Theorem 2. Let $C_{i}$ be a solution. If $C_{i} \in \rho^{m}\left(C_{i-1}\right)$ then there exists such $n$ that $C_{i} \in \rho^{\prime n}\left(C_{i-1}\right),\left(m, n \in \mathbb{N}^{0}\right)$.

Proof (by induction). Let $j_{m}$ be the last literal in $C_{i}$. By Theorem $1 j_{m}$ cannot be a dependent provider ( $C_{i}$ is a legal subsequence) and by Def. $3 C_{i}$ is I/Ocomplete. There are two cases to consider:

1. $j_{m}$ is not a dependent consumer and it exists a macro $b$ s.t. $b=j_{m}$.

2. $j_{m}$ is a dependent consumer and it exists a macro $b$ s.t. $b=j_{i} \ldots j_{m}$.

Basis: Let $n=1$.

Consider the first case $b=j_{m}$. Given that $C_{i} \in \rho^{1}\left(C_{0}\right)$, all the input argument values of $j_{m}$ are + var in the head of $\perp$ (ie. max_prov $\left(j_{m}\right)=0$ ) and $b$ is a legal subsequence given $\emptyset$. Therefore $C_{i} \in \rho^{\prime 1}\left(C_{0}\right)$. Now consider the second case $b=j_{i} \ldots j_{m}$. Since $C_{i} \in \rho^{m}\left(C_{0}\right)$ where $m=\left|C_{i}\right|$, max_prov $\left(j_{i}\right)=0$ and $b$ is a legal subsequence given $\emptyset$. Thus $C_{i} \in \rho^{11}\left(C_{0}\right)$. 
Induction Step: Consider any $n>1$.

Assume again the case when $b=j_{m}$. For $b$ to be a legal subsequence given $C_{i-1}$ it is only required that at least one provider for every input argument of $j_{m}$ is already in $C_{i-1}$. All the providers of $j_{m}$ can be added before $b$ because $\forall$ providers $k$ of $j_{m}, \max _{\text {pprov }}\left(j_{m}\right)>\max \_$prov $(k)$. Then a $\rho^{\prime}$-chain $\left(\rho^{1} \ldots \rho^{\prime n-1}\right)$ can be found so that all the required providers of $j_{m}$ are in $C_{i-1}$ and then $b$ is a legal subsequence given $C_{i-1}$. Thus $C_{i} \in \rho^{\prime n}\left(C_{i-1}\right)$, as claimed.

Assume the second case when $b=j_{i} \ldots j_{m}$. All providers $k\left(k<j_{i}\right)$ of $j_{l}$, $i \leq l \leq m$, are required to be in $C_{i-1}$ so that $b$ is a legal subsequence given $C_{i-1}$ and since max_prov $(b)>\max \_$prov $(k), \forall k$, a $\rho^{\prime}$-chain can be found so that $C_{i-1}$ contains all the required providers of $b$ and $b$ is a legal subsequence given $C_{i-1}$. Hence $C_{i} \in \rho^{\prime n}\left(C_{i-1}\right)$ as claimed.

This completes the proof of the induction step and thus of the theorem.

Using the macro-based $\rho$ the hypothesis space $H^{\prime}$ is reduced to I/O-complete clauses which are legal subsequences of literals, ie. $H^{\prime}:=\{C \subseteq \perp \mid C$ is I/Ocomplete and a legal subsequence\}.

\subsection{Algorithms to Construct the Macros}

The procedure to obtain the ordered set $\mathbf{D}$ of all macros from a given most specific clause and mode declarations can be seen in Figure 1. The algorithm we use to compute the set $\mathbf{A}$ of all the macros starting with a dependent provider $i$ is shown in Figure 2. In the second algorithm, $\times$ means a special case of Cartesian product where the resulting set's elements are numerically ordered; $\mathbf{A}[j]$ represents the element $j$ of set $\mathbf{A}$; and, disaggregate $(\mathbf{A}[j])$ is a function that separates the compound element $\mathbf{A}[j]$ into its component parts.

Example 1. In this example we illustrate how the algorithm in Fig. 2 works. Suppose $\perp=h(+x) \leftarrow p(+x, * y, * z), t(+x,-u), o(* w), q(+x,-w), r(+w,+z)$, $s(+u,+y), m(+z)$, then the algorithm has to compute the set of macros starting with the dependent provider $p(+x, * y, * z)$. Notice that the macros are always ordered according to their position in $\perp$. In this example the ordered set $\mathbf{D}$ is $\mathbf{D}=\{t, q,[p, m],[p, t, s],[p, o, r],[p, q, r],[o, r]\}$

1. $i=p(+x, * y, * z), \mathbf{Z}_{y}=\{s\}, \mathbf{Z}_{z}=\{r, m\}$ then

$\mathbf{A}^{\prime}=\{p\} \times\{s\} \cup\{p\} \times\{r, m\}=\{[p, s],[p, r],[p, m]\}$

for $\mathbf{A}^{\prime}[1]$

(2.1) $\mathbf{Y}_{s,+u}=\{t\}$

(2.2.2) if $\{t\} \cap\{p, s\}=\emptyset$ then $\mathbf{T}_{1}^{\prime}=\{\} \cup\{[p, s]\} \times\{t\}=\{[p, t, s]\}$

(2.2.3) $\mathbf{T}_{1}=\{[p, t, s]\}$

for $\mathbf{A}^{\prime}[2]$

(2.1) $\mathbf{Y}_{r,+w}=\{o, q\}$

(2.2.2) if $\{o, q\} \cap\{p, r\}=\emptyset$ then $\mathbf{T}_{2}^{\prime}=\{[p, o, r],[p, q, r]\}$

(2.2.3) $\mathbf{T}_{2}=\{[p, o, r],[p, q, r]\}$

for $\mathbf{A}^{\prime}[3], \mathbf{T}_{3}=\{[p, m]\}$

2. $\mathbf{A}=\{[p, t, s],[p, o, r],[p, q, r],[p, m]\}$ 
- for every literal $i \in \perp=h \leftarrow 1, \ldots, n$ do:

1. if $i$ has to be a single-literal macro according with Table 2, add $i$ to $\mathbf{D}$.

2 . if $i$ is a dependent provider, construct the set $\mathbf{A}$ of all possible macros starting with $i$ (as shown in Fig. 2) and add $\mathbf{A}$ to $\mathbf{D}$.

- sort $\mathbf{D}$ according to Def. 11.

Fig. 1. Algorithm to obtain the macros

1. let $\mathbf{Z}_{1}, \ldots, \mathbf{Z}_{n}$ be the sets of consumers of $i$ for $* \operatorname{var}_{1}, \ldots, * v a r_{n} \in i$ (ie. $\mathbf{Z}_{j}=\{k \in$ $\perp \mid k$ consumes $\left.\left.* v a r_{j} \in i\right\}\right)$ then: $\mathbf{A}^{\prime}=\{i\} \times \mathbf{Z}_{1} \cup\{i\} \times \mathbf{Z}_{2} \cup \ldots \cup\{i\} \times \mathbf{Z}_{n}$.

2. for every element $\mathbf{A}^{\prime}[j]$ in $\mathbf{A}^{\prime}$ do:

2.1 obtain the providers' set $\mathbf{Y}_{k,+ \text { var }}$ for each +var not provided by $i$ in consumer $k$ of $i$ such that $\mathbf{Y}_{k,+ \text { var }}=\{g \in \perp \mid g$ is a provider of $k$ for + var and $k \in \mathbf{A}^{\prime}[j]$ and $\left.g>i\right\}$.

$\mathbf{2 . 2}$ let $\mathbf{T}_{j}=\left\{\mathbf{A}^{\prime}[j]\right\}$ then for every $\mathbf{Y}_{k,+ \text { var }}$ do:

2.2.1 $\mathbf{T}_{j}^{\prime}=\emptyset$.

2.2.2 $\forall \mathbf{T}_{j}[l]$ if $\mathbf{Y}_{k,+ \text { var }} \cap$ disaggregate $\left(\mathbf{T}_{j}[l]\right)=\emptyset$ then

$\mathbf{T}_{j}^{\prime}=\left\{\mathbf{T}_{j}^{\prime} \cup\left\{\mathbf{T}_{j}[l] \times \mathbf{Y}_{k,+ \text { var }}\right\}\right.$.

2.2.3 $\mathbf{T}_{j}=\mathbf{T}_{j}^{\prime}$.

3. $\mathbf{A}=\bigcup \mathbf{T}_{j}$

Fig. 2. Algorithm to obtain the macros starting with dependent provider $i$

\section{Performance Analysis}

Let us illustrate the search tree reduction obtained with the macros. Assume that $\perp=h \leftarrow 1, \ldots, 16$, that the literals 1 and 4 are dependent providers of the literals 13 and 16, and that 2 and 3 are dependent providers of 14 and 15. Let $\mathbf{D}$ be $\mathbf{D}=\{5,6,7,8,9,10,11,12,[2,14],[2,15],[3,14],[3,15],[1,13],[1$, $16],[4,13],[4,16]\}$. Suppose that we are looking for a two-literal clause. The search-trees expanded by IDA* using both methods are shown in Figure 3.

The main advantage of using the macro-based refinement operator is the reduction of the search space; however, there is a cost for obtaining the macro's set D. To analyze the performance of the macro-based method, we perform experiments on four datasets. The first dataset contains 180 positive and 17 negative examples of valid chess moves for five pieces ${ }^{5}$; the second consists of 3340 positive and 1498 negative examples of "safe" 6 minesweeper moves; the third one is the dataset used in [2] with 256 positive and 512 negative examples of road sections where a traffic problem has occurred; and the last one is the ILP benchmark dataset mutagenesis [12] with 125 positive and 63 negative examples.

Mio was run twice with the same parameters on every dataset: once using the literal-based and once the macro-based method. For the first two datasets we created 5 folds and for the last two 10 folds. Both methods were compared

${ }^{5} E^{+}$of this dataset are contained in the distribution package of CProgol 4.4.

${ }^{6}$ Tiles without a mine. 


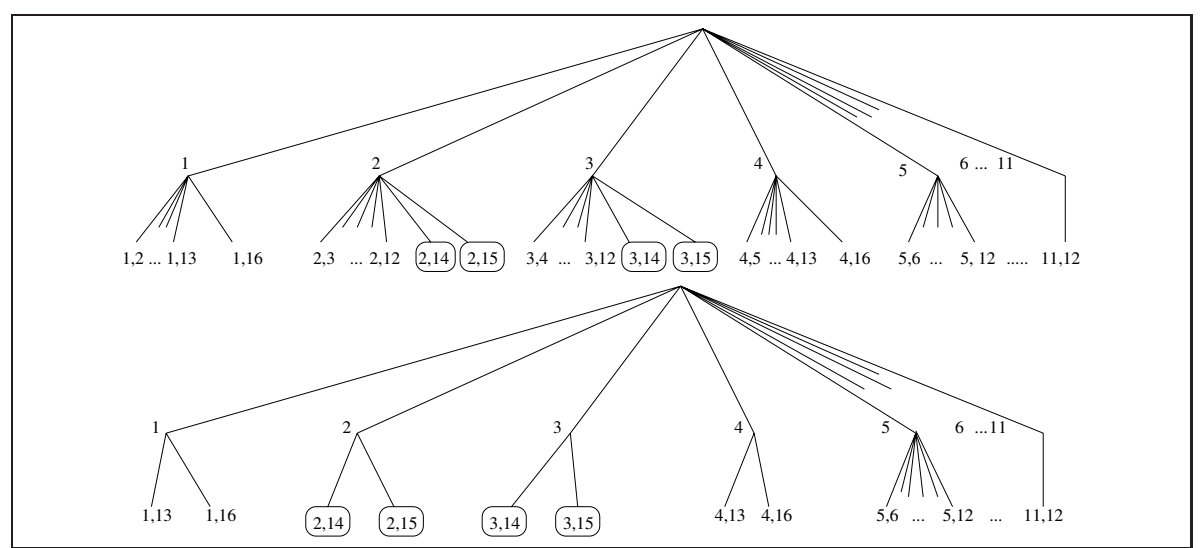

Fig. 3. Search trees expanded by IDA* looking for two-literal clauses. The literalbased method considers 74 leaf-nodes (top) and the macro-based only 36 (bottom). The nodes surrounded by a rectangle are the solutions

using the average number of nodes expanded per search (Avg. N/S) and the average run-time (Avg. RT). For these experiments Mio performs parallel search as described in [9]. The results can be seen in Table 3.

Mio obtained exactly the same theory for chess, minesweeper and mutagenesis using both methods. In the traffic problem, the theory obtained with the macro-based method has an extra literal in one clause. The reason for this is that the literal defined as dependent provider in the traffic problem does not succeed for every input argument value (it is satisfied by $75.22 \%$ of the examples). However, this does not affect the accuracy of the theory.

The macro-based method is in average 8 times faster than the literal-based method. However, the actual speedup on every dataset depends on problem features such as the size of the search space, how long it takes to compute $\operatorname{eval}(C)$, and the number of dependent providers. The macros are suitable for any domain where there exists a dependent provider; if a dependent provider is falsely declared, the macro approach obtains clauses with unnecessary literals.

\section{Related Work}

Mio uses mode declarations to define the hypothesis language and computes $\perp$ in the same way as Progol [7] does. However, Mio differs from Progol in the search

Table 3. Comparison between the literal-based and the macro-based method

\begin{tabular}{|l|c|c|c|c|c|c|c|c|}
\hline Dataset & \multicolumn{2}{|c|}{ Chess } & \multicolumn{2}{c|}{ Minesweeper } & \multicolumn{2}{c|}{ Traffic } & \multicolumn{2}{c|}{ Mutagenesis } \\
\hline Method & $\begin{array}{c}\text { Literal- } \\
\text { based }\end{array}$ & $\begin{array}{c}\text { Macro- } \\
\text { based }\end{array}$ & $\begin{array}{c}\text { Literal- } \\
\text { based }\end{array}$ & $\begin{array}{c}\text { Macro- } \\
\text { based }\end{array}$ & $\begin{array}{c}\text { Literal- } \\
\text { based }\end{array}$ & $\begin{array}{c}\text { Macro- } \\
\text { based }\end{array}$ & $\begin{array}{c}\text { Literal- } \\
\text { based }\end{array}$ & $\begin{array}{c}\text { Macro- } \\
\text { based }\end{array}$ \\
\hline Avg. N/S & 41.56 & 21.55 & 1141.90 & 69.90 & 3179.47 & 543.41 & 58905.4 & 14299.5 \\
Avg. RT & $3.33 \mathrm{~s}$ & $2.43 \mathrm{~s}$ & $16 \mathrm{~h} 33 \mathrm{~m}$ & $51 \mathrm{~m}$ & $3 \mathrm{~h} 17 \mathrm{~m}$ & $29 \mathrm{~m}$ & $7 \mathrm{~h} 45 \mathrm{~m}$ & $1 \mathrm{~h} 46 \mathrm{~m}$ \\
\hline
\end{tabular}


strategy, the heuristics, and the type strictness. Additionally the literal-base $\rho$ differs from Progol's refinement operator in that it does not perform splitting.

Macros were introduced by Korf [5] as a domain-independent weak-method for learning. He cataloged the macro-operators as a non-optimal problem solving technique. Junghanns and Schaeffer [3] used macros to reduce the search-tree for Sokoban while preserving optimality. McGovern and Sutton [6] proposed the use of macros as a method to accelerate reinforcement learning. The macro-based method presented in this work preserves optimality and reduces the search-space of a multirelational learning system.

In multirelational learning the macros can be considered as a general framework for syntactic bias specification such as MOBAL's schemata [4] or relational clichés [11]. Relational clichés add sequences of literals to a clause as a heuristic way to solve the search myopia of a greedy algorithm. Although macros could solve too the shortsightedness of a greedy search, they are not a heuristic but a formal method to reduce the search space that guarantees that the same solutions as the base method are found. Blockeel and De Raedt [1] propose a lookahead technique to improve the evaluation of a clause when a dependent provider is added. In their technique the refinement operator is redefined to incorporate twostep-refinements; the macro-based $\rho$ can be seen as a refinement operator which incorporates n-step-refinements. Contrarily to macros, the lookahead increases the run time and requires the user to provide a template for every providerconsumer match. In contrast to all these methods, in our approach macros are computed automatically and the user only needs to mark the output variables of every dependent provider with the *var notation.

Quinlan included in Foil determinate literals to overcome the greedy search myopia by adding at once all the determinate literals to a clause [10]. The determinate literals are found automatically by Foil; however, since all the determinate literals are added, a clause refining (pruning) step is later needed to remove the unnecessary literals. Determinate literals must be uniquely satisfied by all the positive examples while a dependent provider must be satisfied by all the examples but can be multiple satisfied.

\section{Conclusions and Future Work}

In this paper we propose a downward refinement operator $(\rho)$ which adds macrooperators to a clause. By using this macro-based $\rho$ a reduction in the search space is obtained which results in shorter run-times. We have proved that this refinement operator finds the same solutions as the literal-based one. In addition we present two algorithms to compute automatically a set of macros given a most specific clause and a set of mode declarations. A literal's classification based on the literal's consumer/provider properties is proposed to assist the user to determine whether a literal can be marked as a dependent provider.

For this work we have used as base case a refinement operator similar to the one used in Progol [7]; however, we believe that macro-operators are also suitable for other refinement operators and as a lookahead technique for greedy 
systems. In the current implementation the user has to indicate in the mode declarations which literals are dependent providers; however, it should be possible to perform an automatic analysis of the body literals and determine which ones are dependent providers. It is part of the future work to explore these ideas.

\section{Acknowledgments}

This work was partially supported by a scholarship of the federal state SachsenAnhalt, Germany. We would like to thank Sašo Džeroski for providing the traffic dataset, and O. Meruvia and S. Hoche for helping to improve this paper.

\section{References}

1. Hendrik Blockeel and Luc De Raedt. Lookahead and discretization in ILP. In Sašo Džeroski and Nada Lavrac, editors, Proc. of the rth Int. Workshop on ILP, volume 1297 of Lecture Notes in AI, pages 77-84. Springer-Verlag, 1997. 367

2. Sašo Džeroski, Nico Jacobs, Martin Molina, Carlos Moure, Stephen Muggleton, and Wim Van Laer. Detecting traffic problems with ILP. In D. Page, editor, Proc. of the 8th Int. Conference on ILP, volume 1446 of Lecture Notes in AI, pages 281-290. Springer-Verlag, 1998. 365

3. Andreas Junghanns and Jonathan Schaeffer. Sokoban: A challenging single-agent search problem. In IJCAI Workshop "Using Games as an Experimental Testbed for AI Reasearch", pages 27-36, 1997. 367

4. Jörg-Uwe Kietz and Stefan Wrobel. Controlling the complexity of learning in logic through syntactic and task-oriented models. In S. Muggleton, editor, Inductive Logic Programming, pages 335-359. Academic Press, 1992. 367

5. Richard E. Korf. Macro-Operators: A weak method for learning. Artificial Intelligence, 26(1):35-77, 1985. 358, 367

6. Amy McGovern, Richard S. Sutton, and Andrew H. Fagg. Roles of macro-actions in accelerating reinforcement learning. In Proc. of the Grace Hopper Celebration of Women in Computing, 1997. 367

7. Stephen Muggleton. Inverse entailment and Progol. New Generation Computing Journal, 13:245-286, 1995. 358, 359, 366, 367

8. Shan-Hwei Nienhuys-Cheng and Ronald de Wolf. Foundations of Inductive Logic Programming, volume 1228 of Lecture Notes in AI. Springer-Verlag, 1997. 358

9. Lourdes Peña Castillo and Stefan Wrobel. On the stability of example-driven learning systems: a case study in multirelational learning. In C. A. Coello Coello, A. de Albornoz, E. Sucar, and O. Cairo, editors, Proc. of MICAI'2002, volume 2313 of Lecture Notes in AI. Springer-Verlag, 2002. 358, 359, 366

10. J. Ross Quinlan. Determinate literals in inductive logic programming. In John Mylopoulos and Raymond Reiter, editors, Proc. of the 12th IJCAI, volume 2, pages 746-750. Morgan Kaufmann, 1991. 367

11. Glenn Silverstein and Michael J. Pazzani. Relational clichés: constraining constructive induction during relational learning. In L. Birnbaum and G. Collins, editors, Proc. of the 8th Int. Workshop on Machinge Learning, pages 203-207, 1991. 367

12. Ashwin Srinivasan, Stephen Muggleton, Ross D. King, and Michael J. E. Sternberg. Theories for mutagenicity: a study of first-order and feature based induction. Artificial Intelligence, 85(1-2):277-299, 1996. 365 